\title{
Isolated Talar Dislocation without a Fracture: An Unusual Presentation
}

\author{
Authors \\ Dr Dibakar Ray, Dr Ashok Kumar Chanda, Dr Srikanta Tagore Sarkar, \\ Dr Somnath Tirkey, Dr Ashik Iqubal, Dr Wrrtik Porel
}

\begin{abstract}
Dislocation of the talus from all its surrounding joints (talonavicular, tibiotalar, subtalar) not accompanied by a fracture (talus, navicular, calcaneus, malleoli) is an extremely rare. Management guideline is also not clear. Here cases were treated with closed reduction under general anesthesia with good followup result.

Key Words-Talus, dislocation, avuscular necrosis
\end{abstract}

\section{INTRODUCTION}

Dislocation of the talus from all its surrounding joints (talonavicular, tibiotalar, subtalar) not accompanied by a fracture (talus, navicular, calcaneus, malleoli) is an extremely rare injury. Its exact incidence is unknown ${ }^{[1]}$. Depending on whether the foot comes to supination or pronation the dislocation can be either anterolateral (the most usual) or posteromedial ${ }^{[2]}$. The few case reports found in the literature and the non-existent guidelines add to the confusion regarding the optional method of treatment. Complete dislocation of the talus without an accompanying fracture usually has to do with a young patient at the second to fourth decade of his life. Major complications and in particular infection, avascular necrosis of the talus and post-traumatic arthritis are therefore even more devastating because they affect patients running the most active and demanding period of their lives ${ }^{[3] .}$ Of these complications, infection mainly depends on whether the injury was open or closed. Avascular necrosis though, is depended on the amount of blood supply that remains to a bone which is notorious for its problematic vascularity. We present our method of closed conservative treatment in two cases with closed total talus dislocation without fracture. Whether a closed or open approach should be chosen in order to minimize complications and augment possibilities for a favourable outcome that is of debate!

\section{MATERIAL AND METHODS}

Case I:

A 35-year-old woman was brought to the emergency room because of a road traffic accident. Her left ankle was swollen and the foot was in slight equinus. There was no sensory or motor loss, but initial vascular assessment was difficult because pulses could not be felt and the capillary refill was borderline. X-Rays (Figs.1,2) revealed an antero-lateral dislocation of the talus with no fracture. 

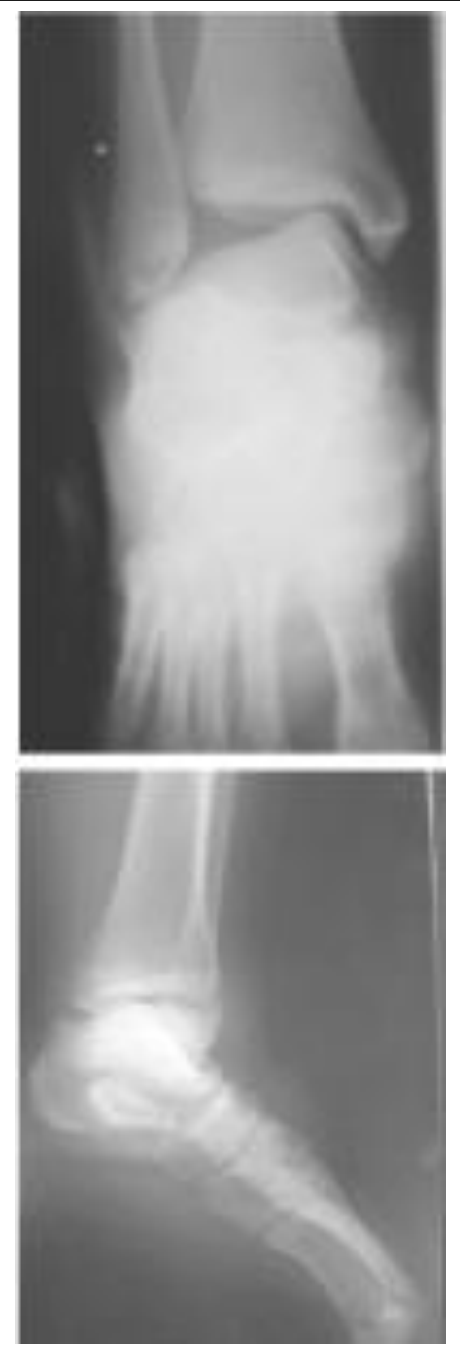

FIGURE-1,2:

Case I, AP and Lat X-Rays of the Rt. ankle reveals complete talar dislocation.

\section{Case II:}

A 45-year-old man was brought to the emergency room because of a motor-bike accident. His major problem was a head injury, but at the same time his left ankle was swollen with a very tensed skin over its lateral side and the foot in slight equinus and supination. Neurological and vascular examination showed no pathological signs. The radiographic examination showed a total anterolateral dislocation of the left talus in all projections (Fig.3) without any accompanying fracture.

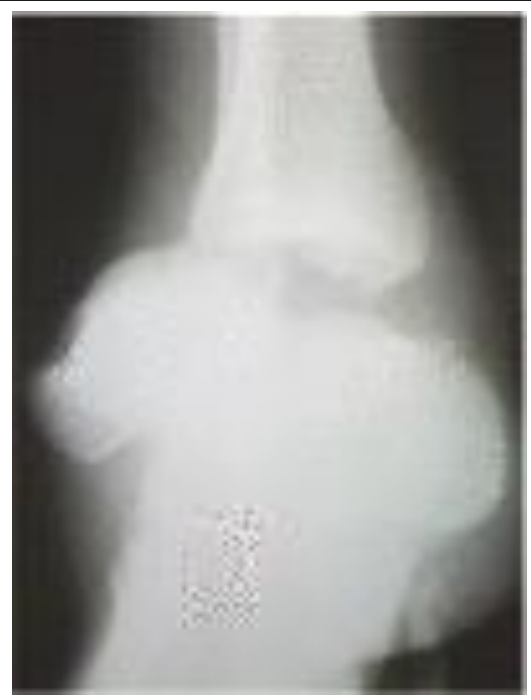

FIGURE: 3

Case II, X-Rays reveal complete dislocation without fracture.

In both cases a closed reduction was performed under general anesthesia. With the knee flexed to relax the posterior leg muscles, traction was applied to the foot and counter-traction to the tibia. The talus was then pushed to a posterior and medial direction. Reduction occurred relatively easily.The reduction was stable with manual manipulation and was confirmed radiologically in the operating room (Figs.4,5,6). After reduction, pulses could be palpated clearly in case I.

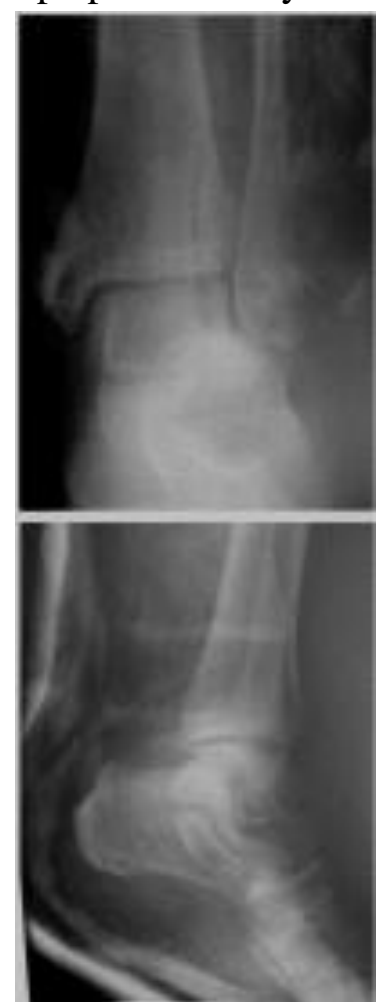

FIGURE: 4,5 
Case I, X-Rays post reduction.

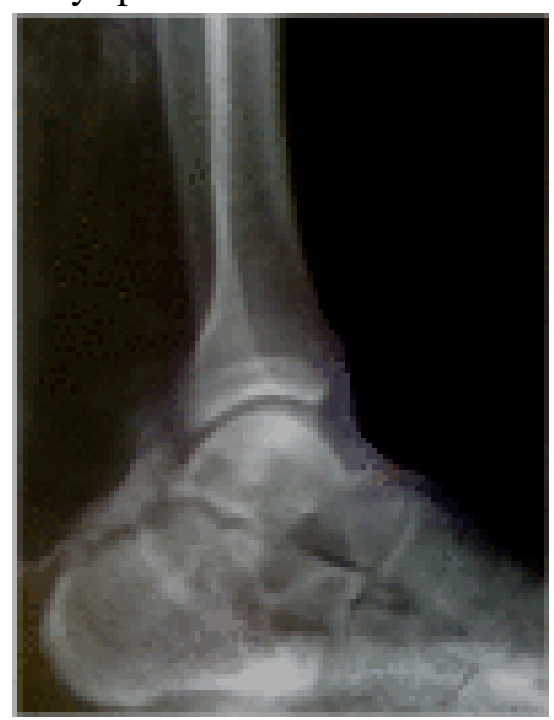

FIGURE: 6

Case II, Lat X-Ray post reduction.

In patient I, a below knee back slab and then full plaster cast was applied for a total of 6 weeks. Then partial weight bearing with the use of a walker for 1 week, 1 week with axillary crutches, 1 week with one crutch and finally full weight bearing and motion was allowed at 9 weeks. The patient was followed up for two years at which time no signs of avascular necrosis or osteoarthritis were detected clinically or radiologically. She had no symptoms, more than $90 \%$ range of motion in the ankle and subtalar joints and no degenerative changes on radiographs, an excellent result according to the criteria of Kenwright and Taylor ${ }^{[4]}$. Patient II was lost from follow-up. It was reported by him over telephone that one year later he was walking with no problems.

\section{DISCUSSION}

For open injuries almost all authors suggest that open reduction is the best choice and talectomy or even tibiocalcaneal fusion should be reserved for later salvage procedures ${ }^{[5,6]}$, even in the situation of a missing talus ${ }^{[7]}$. Ritsema reports two avascular necroses in five cases. According to him, a rapid open reduction for closed injuries is the best choice ${ }^{[8]}$. Korovessis et al. report a pure complete dislocation of the talus, treated with open reduction because of inability for a closed one with good results ${ }^{[9]}$. Taymaz and Gunal ${ }^{[1]}$ reported a case of closed total talus dislocation treated by closed means with an excellent result. Hadji et al. ${ }^{[10]}$ also report a good result after closed reduction of a complete talus dislocation on a three year follow-up.

According to Kenwright and Taylor ${ }^{[4]}$ the incidence of avascular necrosis is depended on the magnitude of displacement of the body of talus. This does make sense because as approximately $60 \%$ of the surface of the talus is covered by articular cartilage, only a limited surface is available to provide adequate vascular perforation [11]. The major blood supply to the body is provided by the artery of the tarsal canal, a branch of the posterior tibial artery. The superior neck branches of the dorsalis pedis artery supply small areas of the body but have been found to have anastomoses with the other arteries. Arterial anastomoses within the bone are numerous ${ }^{[6]}$.

Impressively, there is clinical and radiological (bone scan, MR angiogram) evidence that in cases of complete anterolateral dislocation of the talus vascularity of the bone was maintained by these superior branches through anterior soft tissue attachments. We are therefore led to believe that any open reduction can have a negative influence to the already bad blood supply of the talus ${ }^{[1,2,12]}$. Infection is a complication of open dislocations. Cleaning of the lesion, debridement, antibiotic administration and secondary closure can minimize the risk of an infection ${ }^{[2,7]}$.

Post-traumatic arthritis may occur in any of all the involved joints. The reason can be collapsing of the talus or/and osteochondral fractures of the tarsal bones that could have occurred at the time of injury and were not recognized by plain X-Rays $[1,2,13,15]$. In such cases the use of the CT scan to exclude osteochondral fractures and additionally produce a 3-d image of the dislocation of the talus would help both treatment (planning of the manipulations) and prognosis.

In our case no avascular necrosis or post-traumatic arthritis occurred according to the clinical 
findings, comparative X-Rays with the healthy foot and MRI.

\section{CONCLUSIONS}

Conclusively, literature suggests that in open dislocations of the talus, after an attentive debridement the proper choice should be an open reduction. In closed lesions, it is proposed that a closed reduction under general anaesthesia must be attempted. If this is impossible an open reduction should be the next step. Special attention should be given to avoid injury of the remaining feeding vessels. Talectomy and arthodesis should be reserved as later salvage procedures.

\section{REFERENCES}

1. Taymaz A, Gunal I. Complete dislocation of the talus - Unaccompanied by fracture. J Foot Ankle Surg. 2005;44:156-8.

2. Bas T, Vicent V, Alcantara P, Llabres AJ. Complete dislocation of the talus: a report of 5 cases. Foot.1994;4:102-5.

3. Ries M, Healy WA Jr. Total dislocation of the talus: case report with a 13-year follow up and review of the literature. Orthop Rev. 1988;17:76-80.

4. Kenwright J, Taylor RG. Major injuries of the talus. J Bone Joint Surg. 1970;52B:3648.

5. Huang PJ, Fu YC, Tien YC, et al. Open total talar dislocation - report of two cases. Kaohsiung J Med Sci. 2000;16:2148.

6. Palomo TJM, Cruz RE, Granell BV, Monzonis GJ. Open total talus dislocation: case report and review of the literature. $\mathrm{J}$ Orthop Trauma. 1997;11:45-9.

7. Hiraizumi $\mathrm{Y}$, Hara $\mathrm{T}$, Takahashi $\mathrm{M}$, Mayehiyo S. Open total dislocation of the talus with extrusion (missing talus): report of two cases. J Foot Ankle Surg. 1992;13:473-7.
8. Ritsema GH. Total talar dislocation. J Trauma. 1988;28:692-4.

9. Korovessis P, Spastris P, Sidiropoulos P, Katsardis T, Spyropoulos P. Complete lateral dislocation of the talus without fracture. J Orthop Trauma. 1992;6:392.

10. Hadji M, Galli M, Moalla R, Kmantar L, Hamdi A. Conservative treatmant of talar dislocation: a case report. Rev Chir Orthop Reparatrice Appar Mot. 2004;90:285-8.

11. Detenbeck LC, Kelly PJ. Total dislocation of the talus. J Bone Joint Surg. 1969;51A:283-8.

12. Schiffer G, Jubel A, Elsner A, Andermahr J. Complete talar dislocation without late osteonecrosis: clinical case and anatomical study. J Foot Ankle Surg. 2007;46:120-3.

13. Maffulli N, Lepore L, Francobandiera C, Cifarelli V. Total dislocation of the talus. J Foot Ankle Surg.1989;28 :208-12.

14. Mestdagh H, Duquennoy H, Claisse PR, Sensey JJ, Gougeon F. Long term prognosis of tarsal dislocation. Arch Orthop Trauma Surg. 1982;99:153-9.

15. Pennal GF. Fractures of the talus. Clin Orthop. 1963;30:53-63. 